Jan Jastrzȩbski, Uniwersytet Gdański, Instytut Matematyki, Wita Stwosza

57, 80952 Gda/nsk, Poland. e-mail: jjas@ksinet.univ.gda.pl

\title{
LOCAL CHARACTERIZATION OF FUNCTIONS HAVING THE CANTOR INTERMEDIATE VALUE PROPERTY
}

\begin{abstract}
We find the local characterizations of the Cantor intermediate value property and the strong Cantor intermediate value property. Moreover we show that there is no similar local characterization for the weakly Cantor intermediate value property.
\end{abstract}

The local property of Darboux, the local connectivity and the local almost continuity of a function have been considered by many authors (see [1], [2], $[3]$, [4] and [5]). There arises a question whether it is possible to define a local Cantor intermediate value property at a point in such a way that this property characterizes the global Cantor intermediate value property. We attempt such a definition in this article.

First we introduce some notions and definitions.

Definition 1. We say that a function $f: I \rightarrow \mathbb{R}$ has the Cantor intermediate value property (we write $f \in \mathrm{CIVP}$ ) if for each pair of points $p, q \in I(p<q$ and $f(p) \neq f(q))$ and for each Cantor set $K$ between $f(p)$ and $f(q)$ there exists a Cantor set $C \subset(p, q)$ such that $f(C) \subset K$.

If, additionally, we assume that $\left.f\right|_{C}$ is continuous, then we obtain the definition of the strong Cantor intermediate value property (we write $f \in$ SCIVP).

Definition 2. We say that a function $f: I \rightarrow \mathbb{R}$ has the weak Cantor intermediate value property (we write $f \in \mathrm{WCIVP}$ ) if for all $p, q \in I(p<q$ and $f(p) \neq f(q))$ there exists a Cantor set $C \subset(p, q)$ such that $f(C)$ is contained between $f(p)$ and $f(q)$.

Mathematical Reviews subject classification: 26A15

Received by the editors June 23, 1997 
Clearly

$$
\text { SCIVP } \subset \mathrm{CIVP} \subset \mathrm{WCIVP} .
$$

By $C(f, x), C^{-}(f, x)$ and $C^{+}(f, x)$ we denote the cluster set of a function $f$ at a point $x$, the left cluster set of $f$ at $x$ and the right cluster set of $f$ at $x$, respectively.

Recall that $C(f, x)=C^{-}(f, x) \cup C^{+}(f, x)$.

For any function $f: I \rightarrow \mathbb{R}$, we define a multifunction $F: I \rightarrow \mathbb{R}$ by $F(x)=C(f, x)$ for all $x \in I$.

In the standard way one can prove the next two theorems.

Theorem 1. If a function $f: I \rightarrow \mathbb{R}$ fulfills the conditions

(1) $C(f, x)$ is connected

(2) $f(x) \in C(f, x)$

for each $x \in I$, then $F((p, q))$ is connected for all intervals $(p, q) \subset I$.

Theorem 2. If a function $f: I \rightarrow \mathbb{R}$ has the Cantor intermediate value property or the strong Cantor intermediate value property, then for all $x \in I$ the cluster sets $C(f, x), C^{-}(f, x)$ and $C^{+}(f, x)$ are connected and

$$
f(x) \in C^{-}(f, x) \cap C^{+}(f, x) .
$$

Now we introduce local definitions of the Cantor intermediate value property and the strong Cantor intermediate value property.

Definition 3. We say that a function $f: I \rightarrow \mathbb{R}$ has the left-sided Cantor intermediate value property at a point $x_{o}$ (we write $x_{o} \in \mathrm{CIVP}^{-}(f)$ ) if

1. $f\left(x_{o}\right) \in C^{-}\left(f, x_{o}\right)$

2. for all $a, b \in C^{-}\left(f, x_{o}\right)$, for an arbitrary Cantor set $K \subset(a, b)$ and positive $\varepsilon>0$ there exists a Cantor set $C \subset\left(x_{o}-\varepsilon, x_{o}\right)$ such that $f(C) \subset K$.

In an analogous manner we define the notion of the right Cantor intermediate value property at a point $x_{o}$ (we write $x_{o} \in \mathrm{CIVP}^{+}(f)$ ). The function that has the left and the right Cantor intermediate value property at a point $x_{o}$ is said to have the Cantor intermediate value property at the point $x_{o}$. Then we write $x_{o} \in \operatorname{CIVP}(f)$.

If in the above definitions we assume additionally that $f_{\mid C}$ is continuous than we obtain definitions the left, the right and the bilateral strong 
Cantor intermediate value property at the point $x_{o}$. Then we shall write $x_{o} \in \operatorname{SCIVP}^{-}(f), x_{o} \in \operatorname{SCIVP}^{+}(f)$ and $x_{o} \in \operatorname{SCIVP}(f)$, respectively.

One can observe that if $f$ is continuous at a point $x_{o}$ (from one or both sides), then it has the Cantor intermediate value property and the strong Cantor intermediate value property at $x_{o}$ from the same side.

Now we shall prove that the local and global properties of the Cantor intermediate value property are compatible.

Theorem 3. A function $f: I \rightarrow \mathbb{R}$ has the Cantor intermediate value property if and only if $f$ has the Cantor intermediate value property at every point of the interval I.

Proof. Let us assume that $f$ has the Cantor intermediate value property and suppose that there exists a point $x_{o}$ such that $x_{o} \notin \operatorname{CIVP}^{+}(f)$. It is easy to see that $f\left(x_{o}\right) \in C^{+}\left(f, x_{o}\right)$. Then there exists a Cantor set $\mathrm{K}$ such that

1. $K \subset(a, b) \subset C^{+}\left(f, x_{o}\right)$

2. there exists positive number $\varepsilon$ such that $f(C)$ is not contained in $K$ for every Cantor set $C$ included in the interval $\left(x_{o}, x_{o}+\varepsilon\right)$.

Let $x_{1}, x_{2} \in\left(x_{o}, x_{o}+\varepsilon\right)$ be chosen in such a way that $f\left(x_{1}\right)<\inf K$ and $f\left(x_{2}\right)>\sup K$. Then $K \subset\left(f\left(x_{1}\right), f\left(x_{2}\right)\right)$. Therefore there exists a Cantor set $C \subset\left(x_{1}, x_{2}\right)$ such that $f(C) \subset K$ (because $f$ has the Cantor intermediate value property). A contradiction.

Now we assume that $f$ does not have the Cantor intermediate value property and $x \in \operatorname{CIVP}(f)$ for all $x \in I$. There exist an interval $(x, y) \subset I$ and a Cantor set $K \subset(f(x), f(y))$ such that $f(C) \not \subset K$ for every Cantor set $C \subset(x, y)$.

Let us consider two possibilities.

1. There exists a point $x_{o} \in(x, y)$ such that $\operatorname{Int} C^{+}\left(f, x_{o}\right) \cap K \neq \emptyset$ or Int $C^{-}\left(f, x_{o}\right) \cap K \neq \emptyset$.

2. Int $C^{+}\left(f, x_{o}\right) \cap K=\emptyset$ and $\operatorname{Int} C^{-}\left(f, x_{o}\right) \cap K=\emptyset$ for each $x_{o} \in(x, y)$.

where $\operatorname{Int} A$ denotes the interior of the set $A$.

In the first case (for example let Int $\left.C^{+}\left(f, x_{o}\right) \cap K \neq \emptyset\right)$ there exist points $a, b \in C^{+}\left(f, x_{o}\right)$ such that $K_{1}=(a, b) \cap K$ is a Cantor set. There exists a Cantor set $C \subset\left(x_{o}, y\right)$ such that $f(C) \subset K_{1} \subset K$. A contradiction.

Let us consider the second case. Let $t \in K$ be a point of bilateral accumulation of the set K. By Theorem 1, there is $x_{o} \in f^{-1}(t) \cap(x, y)$. Then $C\left(f, x_{o}\right)=\left\{f\left(x_{o}\right)\right\}$; so $f$ is continuous at $x_{0}$. Let $K_{1}$ be the set of all points of bilateral accumulation of the set $K$. Then $f^{-1}\left(K_{1}\right) \cap(x, y) \subset C(f)$, where 
$C(f)$ denotes the set of all points of continuity of the function $f$. The set $K_{1}$ is a Borel set of the power of continuum, $f^{-1}\left(K_{1}\right) \cap(x, y)$ is a Borel set in $C(f)$ and so $f^{-1}\left(K_{1}\right) \cap(x, y)$ is a Borel set of power of continuum. Then there exists a Cantor set $C \subset f^{-1}\left(K_{1}\right) \cap(x, y)$. So $f(C) \subset K_{1} \subset K$.

In the similar way we can prove the following.

Theorem 4. A function $f: I \rightarrow \mathbb{R}$ has the strong Cantor intermediate value property if and only if $f$ has the strong Cantor intermediate value property at every point of the interval $I$.

Now we start to discuss the problem of local characterization of the WCIVP property. First we may say that a function $f: I \rightarrow \mathbb{R}$ has a (local) property $\mathcal{W}$ at a point $x_{o}$, if for each neighborhood $U$ of the point $x_{o}$ every extension of the function $f_{\mid U}$ has this property at $x_{o}$. We shall write, then $f \in \mathcal{W}\left(x_{o}\right)$.

Let us consider the following example. Let $f: I \rightarrow(0,1)$ be a function from the class WCIVP, which transforms each subinterval $(a, b)$ of $I$ onto the interval $(0,1)$. This function takes on its supremum at no point of the interval $I$. Let $x_{o}$ be a given point from the interval $I$. Consider the function defined by

$$
g(x)= \begin{cases}f(x) & \text { for } x \neq x_{o} \\ 2 & \text { for } x=x_{o}\end{cases}
$$

The function $g$ has also WCIVP property.

Theorem 5. There exists no local property $\mathcal{W}(x)$ which characterizes a property $\mathcal{W}$, i.e. such that $f \in$ WCIVP if and only if $f \in \mathcal{W}(x)$ for each $x \in I$.

Proof. If there exists a local property $\mathcal{W}(x)$ which characterizes functions with WCIVP, then the function $f$ from the above example should have it at each point $x \in I$. Then the function $g$ from the same example would have the property $\mathcal{W}(x)$ at each point, in particular at the point $x_{o}$. It means that the substitution of any value in the place of $f\left(x_{o}\right)$ has no effect on the local property $\mathcal{W}\left(x_{0}\right)$. Let $x_{1}, x_{2}$ be distinct points from the interval $I$. The function $h$ defined by

$$
h(x)= \begin{cases}f(x) & \text { for } x \neq x_{i}, i=1,2 \\ 2 & \text { for } x=x_{1} \\ 3 & \text { for } x=x_{2}\end{cases}
$$

has the property $\mathcal{W}(x)$ at each point of $x \in I$ and it should have the property WCIVP, which is impossible.

Theorem 6. For any function $f: I \rightarrow \mathbb{R}$ the set $\operatorname{CIVP}^{+}(f) \triangle \mathrm{CIVP}^{-}(f)$ is at most countable, where $\triangle$ means the symmetric difference of sets. 
Proof. Since the set of $x \in \mathbb{R}$ at which $C^{-}(f, x) \neq C^{+}(f, x)$ or $f(x) \notin$ $C(f, x)$ is at most countable ([6]), it is enough to consider only the set $A$ of all points of $x \in \mathbb{R}$ at which $f(x)$ belongs to $C(f, x), C^{-}(f, x)=C^{+}(f, x)$ and $x \in \operatorname{CIVP}^{-}(f) \backslash \mathrm{CIVP}^{+}(f)$. Let $A_{n}$ (for each $n \in \mathbb{N}$ ) denote the set of all those points $x$ of $A$ for which $f(x) \in C^{+}(f, x)$ and there exists a Cantor set $K \subset\left[\inf C^{+}(f, x)+\frac{1}{n}, \sup C^{+}(f, x)-\frac{1}{n}\right]$ such that $f(C) \not \subset K$ for every Cantor set $C$ contained in $\left[x, x+\frac{1}{n}\right]$, One can see immediately that $A=$ $\bigcup_{n=1}^{\infty} A_{n}$. Now we shall show that none of the sets $A_{n}$ contains its left-sided points of accumulation. Suppose that there exists a sequence $\left(x_{k}\right)$ and a point $x_{o}$ such that $x_{k} \in A_{n}, x_{k} \rightarrow x_{o}, x_{k}<x_{k+1}<x_{o}$ and $x_{o} \in A_{n}$. Then there is $k_{o} \in N$ such that $\left[\inf C^{+}\left(f, x_{k_{o}}\right)+1 / / n, \sup C^{+}\left(f, x_{k_{o}}\right)-\frac{1}{n}\right] \subset \operatorname{Int} C^{-}\left(f, x_{o}\right)$ and $x_{o} \in\left(x_{k_{o}}, x_{k_{o}}+\frac{1}{n}\right)$.

Let $\varepsilon$ be a positive number for which $\varepsilon<x_{o}-x_{k_{o}}$. Now let $K$ be a Cantor set contained in $\left[\inf C\left(f, x_{k_{o}}\right)+1 / / n, \sup C\left(f, x_{k_{o}}\right)-\frac{1}{n}\right]$. Since $x_{o} \in \mathrm{CIVP}^{-}(f)$, then there exists a Cantor set $C \subset\left(x_{o}-\varepsilon, x_{o}\right)$ for which $f(C) \subset K$. A contradiction.

Thus each of the sets $A_{n}$ is countable, and consequently so is $\mathrm{A}$. This proves that the set $\mathrm{CIVP}^{-}(f) \backslash \mathrm{CIVP}^{+}(f)$ is countable.

Similarly one can prove that the set $\operatorname{CIVP}^{+}(f) \backslash \mathrm{CIVP}^{-}(f)$ is countable, what proves the theorem.

In the same way one can prove the next theorem

Theorem 7. For each function $f: \mathbb{R} \rightarrow \mathbb{R}$ the set $\operatorname{SCIVP}^{+}(f) \triangle \mathrm{SCIVP}^{-}(f)$ is countable.

Theorem 8. For every function $f: \mathbb{R} \rightarrow \mathbb{R}$ the set $\operatorname{CIVP}(f)$ is of the type $G_{\delta}$.

Proof. Let $A=\mathbb{R} \backslash \operatorname{CIVP}(f)$. Let

$$
\begin{gathered}
A_{1}=\operatorname{CIVP}^{+}(f) \triangle \operatorname{CIVP}^{-}(f) \\
A_{2}=\{x \in \mathbb{R}: f(x) \notin C(f, x)\} \cup\left\{x \in \mathbb{R} \backslash \operatorname{CIVP}(f): C^{+}(f, x) \neq C^{-}(f, x)\right\} \\
A_{3}=\mathbb{R} \backslash\left(\mathrm{CIVP}^{+}(f) \cup A_{2}\right) \\
A_{4}=\mathbb{R} \backslash\left(\mathrm{CIVP}^{-}(f) \cup A_{2}\right)
\end{gathered}
$$

We can see that $A=A_{1} \cup A_{2} \cup A_{3} \cup A_{4}$. Of course, the sets $A_{1}$ and $A_{2}$ are countable $([6])$.

Consider the set $A_{3}$. Let $B_{n}$ denote the set of all those points $x$ from $A_{3}$ for which there exists a Cantor set $K \subset[\inf C(f, x)+1 / / n, \sup C(f, x)-1 / / n]$ such that $f(C) \not \subset K$ for each Cantor set $C \subset[x, x+1 / / n]$. Then $A_{3}=$ 
$\bigcup_{n=1}^{\infty} B_{n}$. We shall show that if $x$ is a point of left-sided accumulation of $B_{n}$, then $x \in B_{n+1}$. Let $z \in B_{n}$ satisfy

$$
\begin{gathered}
0<x-z<\frac{1}{n(n+1)}, \\
{[\inf C(f, z)+1 / n, \sup C(f, z)-1 / n] \subset} \\
{\left[\inf C(f, x)+\frac{1}{n+1}, \sup C(f, x)-\frac{1}{n+1}\right] .}
\end{gathered}
$$

Then $(x, x+1 / n+1) \subset(z, z+1 / n)$. Since there exists a Cantor set $K \subset$ $[\inf C(f, z)+1 / n, \sup C(f, z)-1 / n]$ such that $f(C) \not \subset K$, for every Cantor set $C \subset(z, z+1 / n)$, then the Cantor set $K$ is chosen correctly for the point $x$. So $x \in B_{n+1}$. It follows now that the set $A_{3}$ is of the type $F_{\sigma}$.

Similarly one can prove that the set $A_{4}$ is type $F_{\sigma}$ also. Hence the set $A$ is of the type $F_{\sigma}$.

In the same way one can prove the last theorem

Theorem 9. For each function $f: \mathbb{R} \rightarrow \mathbb{R}$ the set $\operatorname{SCIVP}(f)$ is of the type $G_{\delta}$

\section{References}

[1] B. D. Garret, D. Nelms, K. R. Kellum, Characterization of Connected Real Functions, Jber. Deutsch. Math. Ver. 73 (1971), 131-137,

[2] R. Gibson, F. Roush, The Cantor Intermediate Property, Topology Proc. 7 (1982), 55-62,

[3] J. M. Jastrzębski, J. M. Jȩdrzejewski, On Connectivity Points of Darboux Functions, Math. Slovaca 39 No. 4 (1989), 391-405,

[4] J. M. Jastrzȩbski, J. M. Jȩdrzejewski, T. Natkaniec, Points of Almost Continuity of Real Functions, Real Analysis Exch. 16 (1990-1991), 415421 ,

[5] H. Rosen, Connectivity Points and Darboux Points of Real Functions, Fund. Math. 89 (1975), 265-269,

[6] W. H. Young, La symétrie de structure des fonctions réeles, Bull. Sci. Math. 2-e sér.,52 (1928), 265-280. 